Check for updates

Cite this: RSC Adv., 2017, 7, 35827

\title{
Manipulation and analysis of an optofluidic multiphase microlens $\uparrow$
}

\author{
S. Y. Lee, ${ }^{a}$ D. Wee, ${ }^{a}$ J. R. Youn (D) *a and Y. S. Song ${ }^{* b}$
}

An optofluidic microlens was investigated in this study by using a gas-liquid interface, and the underlying physics of the microlens formation was exploited by considering fluid parameters that control the shape of the microlens. A microfluidic device was designed and fabricated to secure a stable multiphasic interface in the channel. A theoretical model based on the coupled Stokes-Cahn-Hilliard equations was proposed to understand the characteristics of the lens formation in the device. The results show that nonlinear flow behavior near the gas-liquid interface affects the interface shape due to the effect of surface tension, and the extent of the lens symmetry is inversely proportional to the product of the capillary number (Ca) and Reynolds number (Re).

Received 29th April 2017

Accepted 11th July 2017

DOI: $10.1039 / \mathrm{c} 7 \mathrm{ra0} 4828 \mathrm{a}$

rsc.li/rsc-advances

In this letter, a tunable optofluidic microlens that utilizes the

\section{Introduction}

Optofluidics, an interdisciplinary research of optics and microfluidics, is being rapidly developed for various applications. ${ }^{1-3}$ The optofluidic platform, in general, employs single or multiple fluidic flows to manipulate or guide light at the microscale. ${ }^{4}$ The optofluidic microlens has emerged as a promising optical component due to its advantages over the solid microlens, e.g., wide tunable range and optically smooth interfaces by means of the hydrodynamic tuning mechanism, the ease in changing optical properties with different lens materials, and the simple fabrication and integration of the device. Recently, a few studies have demonstrated the tunable optofluidic microlens using liquid-liquid ${ }^{5-9}$ or gas-liquid interfaces. ${ }^{10,11}$ They have shown considerable performance in experiments and carried out fluid simulation to analyze the lens shape according to the microchannel geometry or flow conditions. For instance, Seow et al. simulated the stream flow with different aspect ratios of the expansion chamber to obtain an optimum design of the chamber for a stable lens interface. ${ }^{7}$ Rosenauer $e t$ al. predicted the lens shape with symmetric and asymmetric expansion chambers at different flow rate conditions to verify that the fabricated chamber achieved the symmetric lens shape. ${ }^{8}$ However, theoretical studies of the optofluidic microlens generated by the interface formation under the multiphase flow are still limited.

${ }^{a}$ Research Institute of Advanced Materials (RIAM), Department of Materials Science and Engineering, Seoul National University, Seoul 08826, Korea. E-mail: jaeryoun@ snu.ac.kr

${ }^{b}$ Department of Fiber System Engineering, Dankook University, Gyeonggi-do 16890, Korea.E-mail: ysong@dankook.ac.kr

$\dagger$ Electronic supplementary information (ESI) available. See DOI: $10.1039 / \mathrm{c} 7 \mathrm{ra} 04828 \mathrm{a}$ gas-liquid flow in a symmetric expansion chamber is reported. The characteristics of the lens are analyzed by controlling the inlet pressures of the liquid flow and gas flow. Moreover, numerical and theoretical analyses are conducted to figure out the formation mechanism of asymmetric and symmetric lens surfaces and relevant physics governing the interface formation. For the theoretical model, the Stokes-Cahn-Hilliard equation is non-dimensionalized, and material and operational parameters, such as the average flow rate $\left(u_{\text {avg }}\right)$, dynamic viscosity $(\mu)$, density $(\rho)$, surface tension coefficient $(\sigma)$, and characteristic length $(L)$, are analyzed based on the theoretical evaluation. In addition, the lens characteristics such as the lens curvature, thickness, and focal length in the microfluidic device are examined by performing optical observation and ray-tracing simulation. The schematic configuration of the microfluidic setup is shown in Fig. 1.

\section{Numerical and theoretical analyses}

\section{Numerical analysis}

Two-dimensional numerical simulation of the two phase flow is conducted for the numerical modeling of the gas-liquid interface of the lens. The coupled Navier-Stokes-Cahn-Hilliard equations are solved using COMSOL Multiphysics ${ }^{\circledR}{ }^{12-15}$ The two phases are separated by a diffuse interface, and the interface position is tracked by solving two additional transport equations for the phase field. The fluids are assumed to be incompressible. The inertia and gravity effects are neglected for the fluid flow in the microchannel. The surface tension at the gas-liquid interface is considered in the momentum equation as shown below.

$$
0=\nabla \cdot\left[-p \mathbf{I}+\mu\left(\nabla \mathbf{u}+(\nabla \mathbf{u})^{\mathrm{T}}\right]+\mathbf{F}_{\mathrm{st}}\right.
$$


(a)

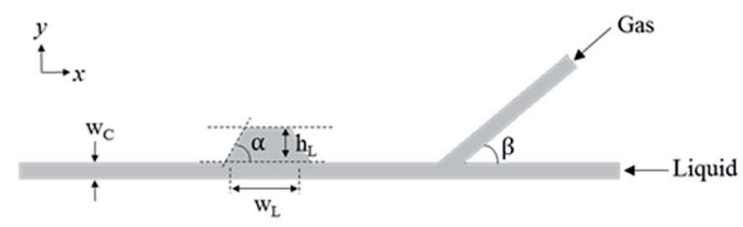

(b)

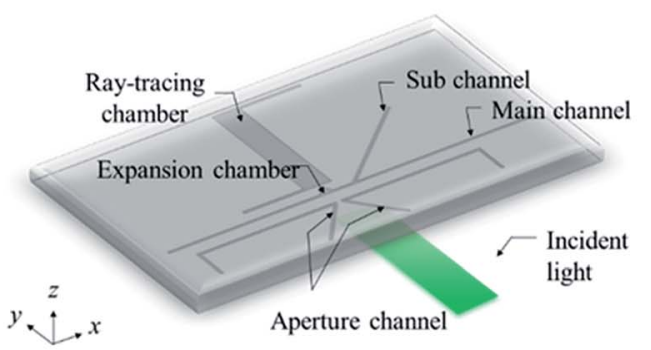

(c)

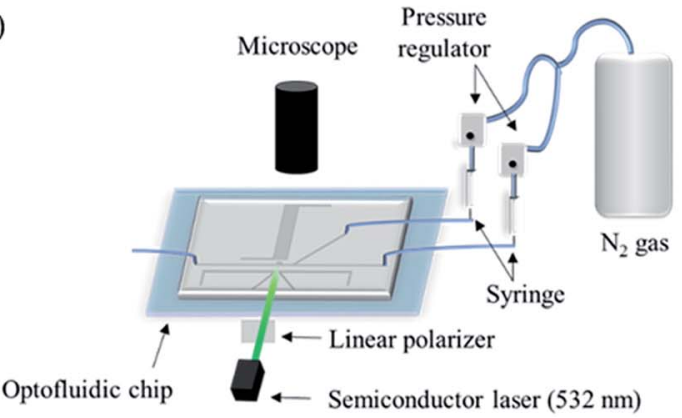

Fig. 1 (a) Schematic illustration of top view of the microchannel used for experiment and simulation. The channel width $\left(w_{C}\right)$ is $200 \mu \mathrm{m}$, the width $\left(w_{L}\right)$ and the height $\left(h_{\mathrm{L}}\right)$ of the expansion chamber are $1000 \mu \mathrm{m}$ and $400 \mu \mathrm{m}$, respectively, and the channel thickness is $25 \mu \mathrm{m}$. The inclination angle of the chamber wall $(\alpha)$ is $60^{\circ}$, and the angle between the main channel and the sub-channel $(\beta)$ is $40^{\circ}$. (b) Schematic configuration of the optofluidic device. (c) Experimental set-up for optical observation.

$$
\begin{gathered}
\mathbf{F}_{\mathrm{st}}=G \nabla \phi \\
G=\lambda\left[-\nabla^{2} \phi+\frac{\phi\left(\phi^{2}-1\right)}{\varepsilon^{2}}\right] \\
\lambda=\frac{3 \varepsilon \sigma}{\sqrt{8}} \\
\frac{\partial \phi}{\partial t}+\mathbf{u} \cdot \nabla \phi=\nabla \frac{\gamma \lambda}{\varepsilon^{2}} \nabla \psi \\
\psi=-\nabla \cdot \varepsilon^{2} \nabla \phi+\left(\phi^{2}-1\right) \phi
\end{gathered}
$$

where $\mathbf{F}_{\mathrm{st}}$ is the surface tension force, $\nabla$ is the del operator, $p$ is the pressure, $\mathbf{I}$ is the identity tensor, $\mu$ is the dynamic viscosity, $\mathbf{u}$ is the flow velocity, $\phi$ is the dimensionless phase field variable, $\psi$ is the phase field help variable, $\lambda$ is the mixing energy density, and $\varepsilon$ is the interface thickness parameter. The Stokes equation is coupled with the convective Cahn-Hilliard equation. The convective Cahn-Hilliard equations track a diffuse interface, which is defined as the region where the phase field $\phi$ is varied from -1 to 1 . The dynamic viscosity is determined by the calculated $\phi$.

\section{Theoretical analysis}

Theoretical analysis is carried out to provide in-depth understanding of the multiphasic microlens formation. The Stokes equation and the convective Cahn-Hilliard equation are nonlinear due to the surface tension force term $\mathbf{F}_{\text {st }}$ and the inertia term $\mathbf{u} \cdot \nabla \phi$ respectively. The characteristics of the microlens is determined by the combination of the non-linear eqn (1) and (5). Since the value of $\phi$ of the convective CahnHilliard equation interactively changes the surface tension force of the Stokes equation, the microlens formation is affected. Basically, the shape and position of the lens interface are determined from the hydrodynamic force balance of fluid. In this regard, the Stokes equation is non-dimensionalized using the dimensionless differential operator and variables: $\nabla^{*}=L \nabla$, $p^{*}=\left(p-p_{\infty}\right) /\left(p_{0}-p_{\infty}\right)$, and $\mathbf{u}^{*}=\mathbf{u} / u_{\text {avg }}$.

$$
\begin{aligned}
0= & -\frac{p_{0}-p_{\infty}}{L} \nabla^{*} p^{*}+\frac{\mu u_{\mathrm{avg}}}{L^{2}} \nabla^{* 2} \mathbf{u}^{*} \\
& +\frac{3 L \sigma}{\sqrt{8}}\left[-\frac{1}{L^{2}} \nabla^{* 2} \phi+\frac{\phi\left(\phi^{2}-1\right)}{L^{2}}\right] \frac{1}{L} \nabla^{*} \phi
\end{aligned}
$$

where $L$ is the characteristic length, $p_{0}-p_{\infty}$ is the reference pressure difference and $u_{\text {avg }}$ is the average flow velocity. Eqn (7) still has a dimension of $m L^{-2} t^{-2}$, where $m, L$ and $t$ represent the mass, length, and time, respectively. Therefore, the equation is multiplied by $L \rho^{-1} u_{\text {avg }}{ }^{-2}$, and $\rho$ was replaced with $\mu / v$. The final non-dimensionalized equation is as follows.

$$
\begin{aligned}
0= & -[\mathrm{Eu}] \nabla^{*} p^{*}+\left[\frac{1}{\mathrm{Re}}\right] \nabla^{* 2} \mathbf{u}^{*} \\
& +\frac{3}{\sqrt{8}}\left[\frac{1}{\mathrm{Ca}}\right]\left[\frac{1}{\mathrm{Re}}\right]\left[-\nabla^{* 2} \phi+\phi\left(\phi^{2}-1\right)\right] \nabla^{*} \phi
\end{aligned}
$$

where the dimensionless numbers, the Euler number (Eu), Reynolds number (Re), and capillary number (Ca), are expressed as $\mathrm{Eu}=\left(p_{0}-p_{\infty}\right) / \rho u_{\text {avg }}^{2}, \mathrm{Re}=\rho u_{\text {avg }} L / \mu$, and $\mathrm{Ca}=$ $\mu u_{\text {avg }} / \sigma$, respectively. As mentioned above, the last term of eqn (8) offers the nonlinearity of the Stokes equation. Thus, the nonlinear phenomena of multiphase fluid, such as asymmetry and singularity, rely upon $\mathrm{Ca}$ and Re. This implies that the resulting nonlinearity decreases as product of $\mathrm{Ca}$ and $\mathrm{Re}$ increases. It is, therefore, expected that a liquid with a high product value of $\mathrm{Ca}$ and $\mathrm{Re}$ would generate a symmetric microlens interface in the symmetric expansion chamber.

\section{Experimental section}

\section{Design and fabrication of the optofluidic chip}

An optofluidic device was fabricated using a soft-lithography process with polydimethyl-siloxane (PDMS, Sylgard 184, Dow Corning, Midland, U.S.A.). A detailed process of fabricating a master is explained elsewhere, ${ }^{9}$ and a cured PDMS replica from the master was attached to the glass substrate by corona treatment. The device consisted of a flow channel, aperture channels, and a ray-tracing chamber. The flow channel was 
Table 1 Material properties of $\mathrm{N}_{2}$, IPA, BA, EG, and PDMS

\begin{tabular}{lllll}
\hline & Property & & & \\
\cline { 2 - 5 } & $\begin{array}{l}\text { Dynamic } \\
\text { viscosity } \\
\left(\mu, \times 10^{-3} \mathrm{~Pa} \mathrm{~s}\right)\end{array}$ & $\begin{array}{l}\text { Density } \\
\left(\rho, \mathrm{kg} \mathrm{m}^{-2}\right)\end{array}$ & $\begin{array}{l}\text { Surface } \\
\text { tension } \\
\left(\sigma, \mathrm{mN} \mathrm{m}^{-1}\right)\end{array}$ & $\begin{array}{l}\text { Refractive } \\
\text { index }(n)\end{array}$ \\
\hline $\mathrm{N}_{2}$ & 0.01747 & 1.2754 & - & 1.0003 \\
$\mathrm{IPA}$ & 1.96 & 786 & 23 & 1.3776 \\
$\mathrm{BA}$ & 5.474 & 1044 & 39 & 1.5396 \\
EG & 16.2 & 1110 & 47 & 1.43 \\
PDMS & - & - & - & 1.4
\end{tabular}

composed of a main channel, sub-channel, and symmetric expansion chamber as shown in Fig. 1(b).

\section{Materials}

The aperture channels were filled with black ink, and a fluorescent dye solution, $3 \mu \mathrm{mol} \mathrm{L^{-1 }}$ of rhodamine B (SigmaAldrich) dissolved in ethylene glycol, was used for the raytracing chamber to observe the ray-path. In the experiments, isopropyl alcohol (IPA) and nitrogen gas $\left(\mathrm{N}_{2}\right)$ were injected through the main channel and sub-channel, respectively, to form the symmetric microlens in the expansion chamber. Two other liquids, benzyl alcohol (BA) and ethylene glycol (EG), were also tested as the main fluid to find a proper lens material, but both liquids showed an asymmetric lens shape. The material properties are listed in Table 1.

\section{Experimental set-up}

The inlet pressure of the main fluids $\left(P_{\text {main }}\right)$ was varied from 20 $\mathrm{kPa}$ to $26 \mathrm{kPa}$, whereas that of the sub-fluid $\left(P_{\text {sub }}\right)$ was fixed at 20 $\mathrm{kPa}$ using a digital pressure regulator (DPG8001-60, OMEGA, Stamford, U.S.A.). The light source was a semiconductor laser (PGL-FS-532, CNI Optoelectronics Technology CO., Ltd, Jilin, China) with a $532 \mathrm{~nm}$ wavelength, and a dichroic linear polarizer was employed for polarization of the light. The lens interface and ray-path were observed using optical microscopy. ${ }^{9}$

\section{Results and discussion}

\section{Shapes of the gas-liquid interfaces}

The lens shape was examined experimentally and numerically for three different liquids as shown in Fig. 2. The main stream and sub-stream flowed in the left-hand direction. It was found that the experimental and numerical results were in good agreement for the three liquids. The interface between the streams of $\mathrm{N}_{2}$ and IPA was nearly symmetric but the interfaces between the streams of $\mathrm{N}_{2}$ and BA, and of $\mathrm{N}_{2}$ and EG were asymmetric. The asymmetry of the interface also increased in the order of IPA, BA, and EG at all the pressure levels. The lens thickness increased as the pressure applied to the main fluid increased. Generation of the symmetric lens is of great importance to secure a high performance. Furthermore, it is essential to fabricate a microlens with a longitudinally tunable focal
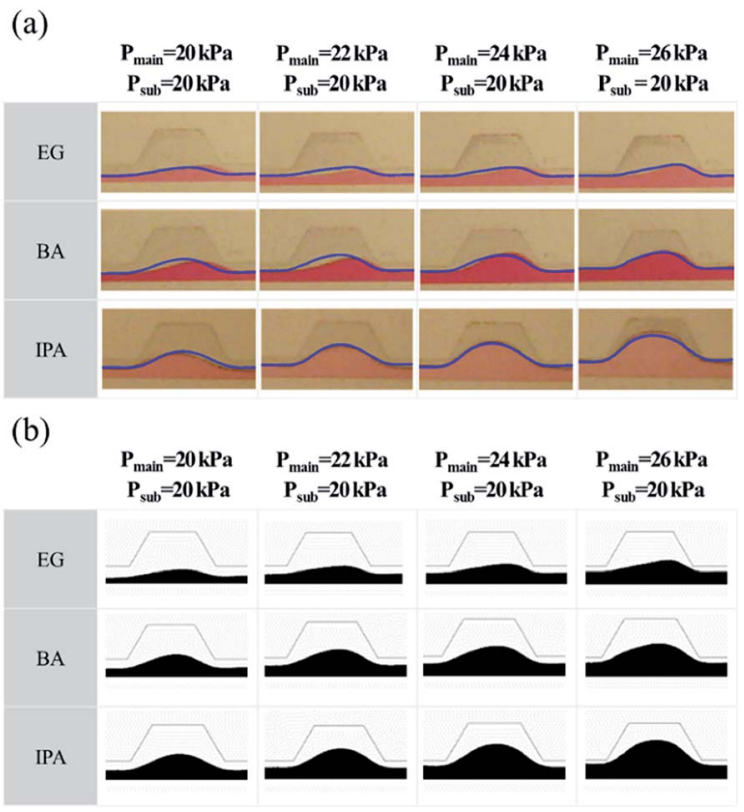

Fig. 2 Images of the gas-liquid interfaces for three liquids at different pressures: (a) microscopic images and (b) simulation results. The simulated interfaces (blue curves) are superposed onto the experimental microscopic images for comparison.

length. For these reasons, IPA was selected in this study as the main liquid to form the symmetric microlens.

\section{Non-linear surface tension effect on the lens shape}

To understand the lens formation in a more systematic manner, the asymmetry of lens was evaluated using the dimensionless numbers presented above. $\mathrm{Ca}$ and Re values were calculated from the experimental conditions for the three liquids at each pressure level. It was inferred that the product value of $\mathrm{Ca}$ and Re significantly affected the shape formation of the lens. Fig. 3(a) shows the measured average velocities used for the calculation. The dimensionless numbers, $\mathrm{Ca}$ and Re, were found quite small at all the pressure levels as presented in Fig. 3(b) and (c). The small values of $\operatorname{Re}(\operatorname{Re} \ll 1)$ indicate the laminar flow in the microchannel (Fig. 3(b)), and the small values of $\mathrm{Ca}(\mathrm{Ca} \ll 1)$ for all the liquids imply that the surface tension effect is dominant over the viscous effect at the gasliquid interface (Fig. 3(c)). ${ }^{16}$ The results of $\mathrm{Ca} \times$ Re are plotted in Fig. 3(d). It was shown that IPA offered the most symmetric lens shape with the largest value of $\mathrm{Ca} \times \mathrm{Re}$ in all the pressure levels, which could explain the lens shape obtained both experimentally and numerically. In short, as the product value of $\mathrm{Ca}$ and Re increases, the interface becomes more symmetric. This quantitative analysis revealed that the $\mathrm{Ca} \times \mathrm{Re}$, i.e., $\rho u_{\mathrm{avg}}{ }^{2} L / \sigma$ acts as a key factor for determining the shape of microlens in the microfluidic device. Since the $\mathrm{Ca} \times \mathrm{Re}$ depends on the dynamic viscosity and $u_{\text {avg }}$ is a function of the viscosity, the shape formation of the lens is affected by the fluid parameters, such as the density, dynamic viscosity, and surface tension of the main fluid as well as the imposed pressure. To the best of 


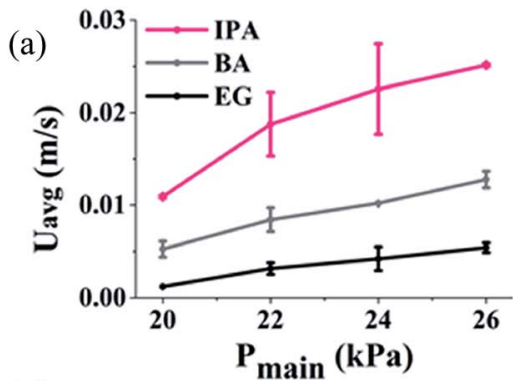

(c)

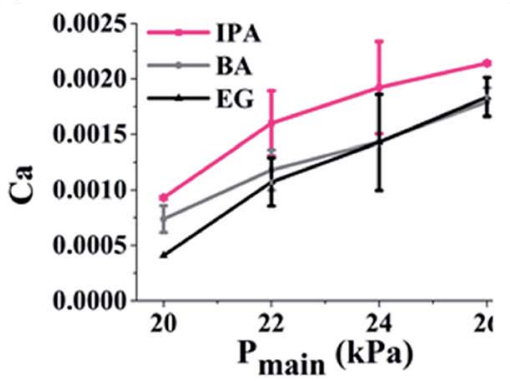

(b)

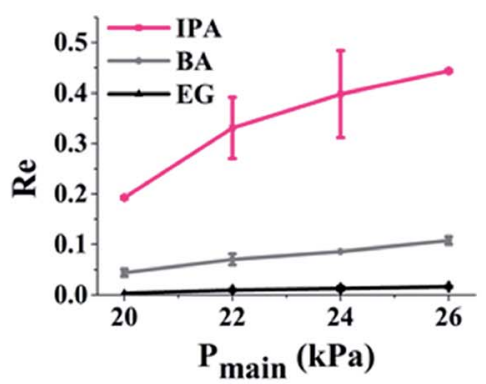

(d)

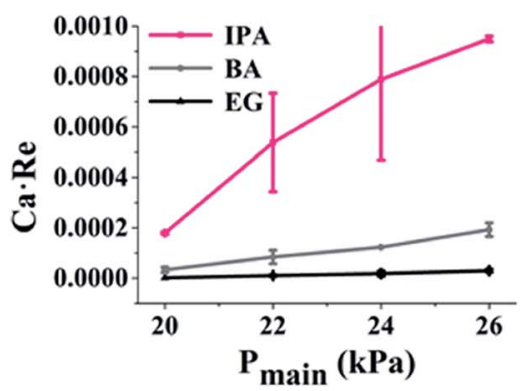

Fig. 3 (a) Measured average velocities, (b) Reynolds numbers, (c) capillary numbers, and (d) product values of capillary numbers and Reynolds numbers for three liquids with respect to pressures.

our knowledge, this is the first report to explore the shape formation of optofluidic microlens in the microfluidic channel by considering quantitative theoretical and numerical evaluation.

\section{Characteristics of the microlens}

The hydrodynamic microlens formed by the streams of $\mathrm{N}_{2}$ and IPA was characterized through optical evaluation. The tunability of the lens was analyzed using the ray-path in fluorescence images in the expanded range of the inlet pressure from $16 \mathrm{kPa}$ to $26 \mathrm{kPa}$ to see the available pressure range, as presented in Fig. 4(a). The focal length $(f)$ was defined as the distance between the flat bottom line of the expansion chamber and the focal point. The focal length decreases with increasing $P_{\text {main }}$ until $P_{\text {main }}$ becomes $24 \mathrm{kPa}$. Therefore, it turns out that the proper pressure range for the focus tuning is between $16 \mathrm{kPa}$ and $24 \mathrm{kPa}$. Fig. 4(b) shows the gray-scaled images of the unfocused beam and focused beam, and Fig. 4(c) presents the light intensity profiles at the positions indicated in the grayscaled images. The light intensity is zero outside of the positions from $-0.2 \mathrm{~mm}$ to $0.2 \mathrm{~mm}$ for the unfocused beam (A, B), which indicates that the apertures completely block the incident light.

The enhancement factor, defined as the ratio of the peak intensity of a focused beam $\left(\mathrm{B}^{\prime}\right)$ to the intensity of an unfocused beam (B), is measured to be $4.07 .{ }^{6}$ The characteristics of the optofluidic lens obtained from the experimental and numerical results are illustrated in Fig. 5. The curvature $(1 / R)$ and the lens thickness $(t)$ were determined by fitting a circle to the gas-liquid interface acquired from the experiment and simulation. The curvature has a maximum value at $P_{\text {main }}=24 \mathrm{kPa}$, and then declines as the pressure increases because the lens interface approaches the wall of the expansion chamber at above $24 \mathrm{kPa}$ (see Fig. S1† in the ESI). ${ }^{6,9}$ The experimental results showed that the tunable focal length between $1.60 \mathrm{~mm}$ and $3.15 \mathrm{~mm}$ could be manipulated in the microfluidic device fabricated in this study, as presented in Fig. 5(c). A ray-tracing code was developed

(a)

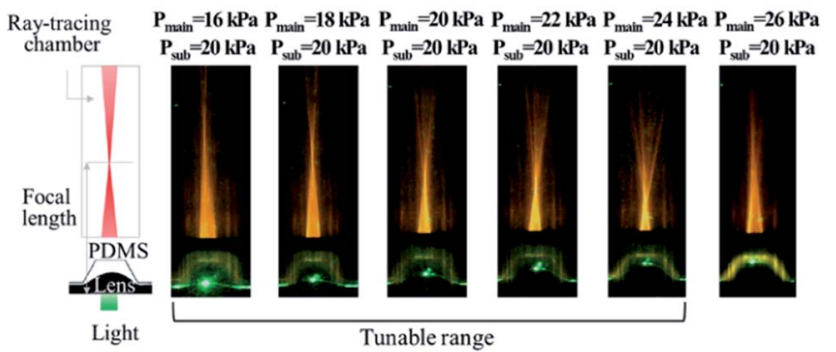

(b)

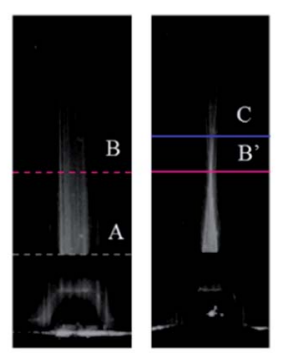

(c)

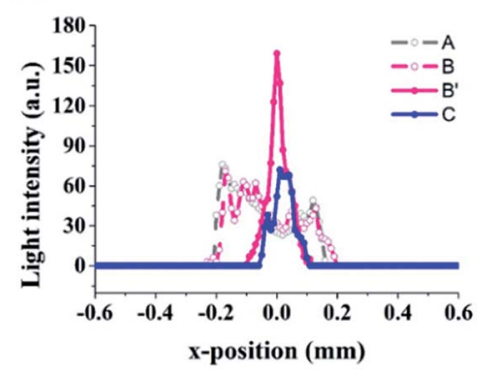

Fig. 4 (a) Schematic illustration of the ray-path observation and fluorescence images of the ray paths at different pressures. (b) Grayscaled images of ray paths. The pressures applied for the main stream and sub-stream were all $20 \mathrm{kPa}$ for the focused beam. The dashed and solid lines indicate the positions where light intensity profiles were evaluated. (c) Light intensity profiles evaluated along the lines. The dashed lines ( $A$ and $B$ ) and solid lines $\left(B^{\prime}\right.$ and $C$ ) indicate the light intensity profile for unfocused beam and focused beam, respectively. 
(a)

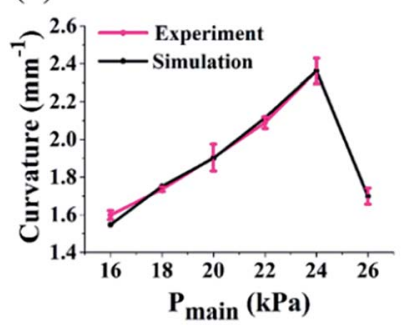

(b)

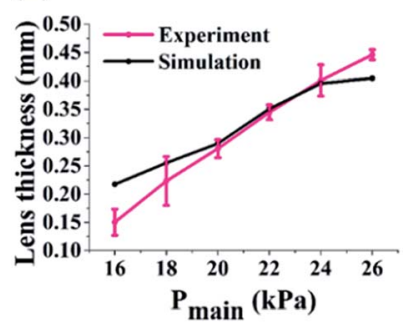

(c)

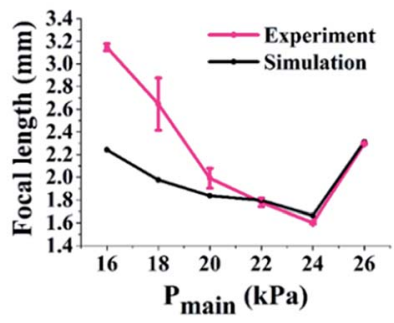

Fig. 5 Comparison of the lens characteristics obtained from simulation and experimental results: (a) curvatures, (b) lens thickness, and (c) focal lengths as a function of the pressure of mainstream.

based on MATLAB ${ }^{\circ}$, and the curvature and lens thickness obtained from the simulation results were employed to predict the focal length numerically (see Fig. $\mathrm{S} 2 \dagger$ in the ESI). The overall trends of the experimental and simulation results were similar, but a slight discrepancy was observed in the case of the focal length at lower inlet pressure because aspherical microlens shape was obtained in the experiment unlike the spherical shape calculated in the simulation. The aspherical microlens leads to decrease in the refraction of light and then increase in the focal length (see Fig. S1 and S3† in the ESI).

\section{Conclusions}

In the current study, various shapes of the microlens based on the gas-liquid interface were investigated from the experimental and numerical results, and the lens formation in the microfluidic device was analyzed by a theoretical model. The Stokes-Cahn-Hilliard equation is non-dimensionalized, and the effect of liquid properties on the lens shape was evaluated. The non-linearity of the surface tension needs to be considered for manipulating the gas-liquid flow in a controlled way in that the non-linear surface tension effect becomes dominant at the gas-liquid interface, especially in microfluidics (or optofluidics). ${ }^{17}$ Our theoretical analysis demonstrated that the interface shape of the lens was determined by the fluid properties as well as the inlet pressure applied to the microfluidic device. In addition, the microlens based on the gas-liquid interface has an advantage of a relatively short focal length due to the large distinction of the refractive indices across the interface, which is critical for the real application in optofluidics. ${ }^{18,19}$ It is expected that this study will provide a way for manipulating the multiphasic microlens based on the comprehensive understanding on the lens formation within the microfluidic device.

\section{Conflict of interest}

There are no conflicts of interest to declare.

\section{Acknowledgements}

This work was supported by the Commercializations Promotion Agency for R\&D Outcomes (COMPA) funded by the Ministry of Science, ICT and Future Panning (MISP). In addition, it was supported by the Industrial Strategic Technology Development Program funded by the Ministry of Trade, Industry and Energy (MI, Korea) [10052641]. The authors are grateful for the support.

\section{References}

1 Y. Yang, A. Liu, L. Chin, X. Zhang, D. Tsai, C. Lin, C. Lu, G. Wang and N. Zheludev, Nat. Commun., 2012, 3, 651.

2 C. Monat, P. Domachuk and B. Eggleton, Nat. Photonics, 2007, 1, 106-114.

3 N.-T. Huang, H.-l. Zhang, M.-T. Chung, J. H. Seo and K. Kurabayashi, Lab Chip, 2014, 14, 1230-1245.

4 X. Zeng and H. Jiang, J. Phys. D: Appl. Phys., 2013, 46, 323001.

5 X. Mao, J. R. Waldeisen, B. K. Juluri and T. J. Huang, Lab Chip, 2007, 7, 1303-1308.

6 S. K. Tang, C. A. Stan and G. M. Whitesides, Lab Chip, 2008, 8, 395-401.

7 Y. Seow, A. Liu, L. Chin, X. Li, H. Huang, T. Cheng and X. Zhou, Appl. Phys. Lett., 2008, 93, 084101.

8 M. Rosenauer and M. J. Vellekoop, Lab Chip, 2009, 9, 10401042.

9 D. Wee, S. Hwang, Y. Song and J. Youn, Soft Matter, 2016, 12, 3868-3876.

10 L. Dong and H. Jiang, Appl. Phys. Lett., 2007, 91, 041109.

11 J. Shi, Z. Stratton, S.-C. S. Lin, H. Huang and T. J. Huang, Microfluid. Nanofluid., 2010, 9, 313-318.

12 https:/www.comsol.com/model/download/318501/models. cfd.capillary_filling_pf.pdf.

13 H. Tian, J. Shao, Y. Ding, X. Li and H. Liu, Langmuir, 2013, 29, 4703-4714.

14 C. Y. Lim and Y. C. Lam, Microfluid Nanofluid, 2014, 17, 131148.

15 P. K. Mondal, D. DasGupta and S. Chakraborty, Phys. Rev. E: Stat. Phys., Plasmas, Fluids, Relat. Interdiscip. Top., 2014, 90, 013003.

16 M. De Menech, P. Garstecki, F. Jousse and H. Stone, J. Fluid Mech., 2008, 595, 141-161.

17 J.-M. Fullana, Y. Ling, S. Popinet and C. Josserand, arXiv preprint arXiv:1507.04329, 2015.

18 P.-T. Lin, H.-Y. Chu, T.-W. Lu and P.-T. Lee, Lab Chip, 2014, 14, 4647-4652.

19 J. Berthelot, S. S. Acimovic, M. L. Juan, M. P. Kreuzer, J. Renger and R. Quidant, Nat. Nanotechnol., 2014, 9, 295-299. 